\title{
GESTIÓN DE LA INFORMACIÓN Y SU EVALUACIÓN EN LOS GABINETES DE COMUNICACIÓN MUNICIPAL
}

\section{Concepción Campillo-Alhama}

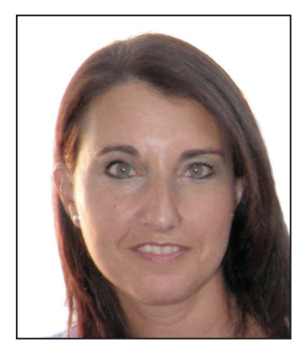

Concepción Campillo-Alhama es licenciada en ciencias de la información por la Universidad Complutense de Madrid y doctora en sociología por la Universidad de Alicante (UA). Es Premio Blas Infante 2010 de Estudio e Investigación sobre Administración y Gestión Pública concedido por el Instituto Andaluz de Administración Pública (IAAP) en su XIV edición. Profesora del Departamento de Comunicación y Psicología Social de la UA, donde imparte docencia sobre estrategia de la publicidad y las relaciones públicas, y gestión de eventos creativos e innovadores. Sus líneas de investigación se centran en la comunicación pública y la comunicación de proximidad de las organizaciones municipales.

http://orcid.org/0000-0001-9886-0049

Universidad de Alicante, Ciencias Sociales, Área de Comunicación Audiovisual y Publicidad Campus San Vicente del Raspeig, Apartado 99. 03080 Alicante, España concepcion.campillo@ua.es

\section{Resumen}

La gestión de la información resulta de especial trascendencia en las instituciones municipales, ya que los logros del equipo de gobierno se trasladan a los ciudadanos a través de mensajes diversos que se identifican como comunicación pública. Las estructuras comunicativas municipales resultan imprescindibles en dichos procesos, destinados a configurar una percepción positiva de la acción de gobierno. Son muy escasas las iniciativas que se han propiciado por parte de estas estructuras para examinar el tratamiento de los hechos informativos en los medios de comunicación. Se presenta un instrumento que permite analizar la agenda mediática en prensa sobre asuntos municipales a partir de la gestión política y administrativa de los ayuntamientos, en un contexto en el que a las funciones estandarizadas de los gabinetes de comunicación, se añaden otras vinculadas a la gestión y evaluación de la información.

\section{Palabras clave}

Información, Documentación, Evaluación, Gestión de información, Ayuntamiento, Comunicación, Estructuras administrativas.

Title: Information management and its evaluation in local government communications

\begin{abstract}
The management of information is of special importance in municipal institutions because citizens hear about their local government's achievement of political objectives through messages identified as public communication. The municipal communication structures are essential in these processes, which are designed to shape positive perceptions of government action. However, these structures have undertaken very few initiatives to examine the treatment of news events in the press. In this paper we present a tool to analyze the media agenda concerning municipal affairs, based on political and administrative management of municipalities, in a context in which the management and evaluation of information is added to the standard functions of the communications office.
\end{abstract}

\section{Keywords}

Information, Documentation, Evaluation, Information management, Municipalities, Town hall, City council, Communication, Administrative structures.

Campillo-Alhama, Concepción (2013). “Gestión de la información y su evaluación en los gabinetes de comunicación municipal". El profesional de la información, noviembre-diciembre, v. 22, n. 6, pp. 515-521.

http://dx.doi.org/10.3145/epi.2013.nov.03

\section{Introducción}

Las organizaciones municipales son instituciones formadas por recursos humanos, materiales, técnicos y sistemas de información que conectan las áreas, departamentos, servicios y negociados integrados en tales estructuras políticas y administrativas. En este contexto, la gestión de la infor- mación y su documentación resulta determinante para la eficacia de los procedimientos a los que se ven sometidos de manera recurrente los ayuntamientos. Las instituciones locales se encuentran supeditadas, en este sentido, no sólo a la propuesta programática realizada por el equipo de gobierno durante los últimos comicios electorales, sino a las características del municipio, su comunidad local, al contex- 
to social, político y económico, así como a las competencias propias, delegadas y compartidas con otras administraciones (Campillo-Alhama, 2010; Campillo-Alhama; RamosSoler, 2013).

Los procesos administrativos y la gestión interna de la información son variables de especial trascendencia, ya que la consecución de los objetivos políticos por parte del equipo de gobierno se traslada a los ciudadanos a través de mensajes de diversa índole (Galán-Galán, 2000). Las estructuras comunicativas municipales actúan como subsistemas imprescindibles en los procesos de comunicación que emergen de las unidades administrativas centralizadas y descentralizadas, para configurar en los ciudadanos una percepción positiva de la acción de gobierno municipal.

No gestionar de forma eficiente la información de la entidad conlleva serios riesgos para su imagen, ya que serán los propios medios de comunicación a través de otras fuentes informativas (con intereses contrapuestos a los de la organización) los que, lanzando mensajes de diversa naturaleza, se conviertan en artífices de procesos de formación de opinión pública no controlados por la fuente objeto de información. Podemos afirmar que, dentro de la estrategia de comunicación externa de cualquier organización pública, la función de mayor incidencia por su repercusión, es la gestión de las relaciones informativas (Almansa-Martínez, 2011; Xifra, 2011).

A pesar de que existen numerosas contribuciones dedicadas al estudio de la producción informativa (Piñuel-Raigada, 1997), son escasas las iniciativas que se han propiciado hasta la fecha desde los gabinetes de comunicación para examinar la eficacia de la producción de documentos propios y su transformación en hechos informativos.

En esta contribución se presenta un instrumento que permite evaluar la agenda mediática en prensa sobre asuntos municipales, a partir de la gestión ejecutada por las diversas estructuras de los ayuntamientos, como entidades declarantes. Tras identificar las variables fundamentales para gestionar y evaluar diferentes aspectos sobre los temas que atañen al municipio, se diseña una base de datos que permite generar un conocimiento exhaustivo sobre cómo la prensa local interpreta tales hechos y los valora a partir de su tratamiento.

\section{El gabinete de comunicación municipal como centro de información y documentación}

Mediante la información periodística se interpreta permanentemente y de forma sucesiva la realidad social (Edo, 2009; Almansa-Martínez, 2011). Tal y como apunta Cebrián (2012), en esta constante interpretación de la realidad, los medios se ven abocados a una selección permanente de las informaciones de las que disponen a través de fuentes comunes, como agencias de información, gabinetes de prensa o contactos personales de los propios periodistas del medio, hasta convertir los hechos municipales, modelados convenientemente, en verdaderos productos informativos.

No se pretende describir la vorágine cotidiana en la que se encuentran inmersos tales departamentos, pero sí interesa apuntar algunas funciones estandarizadas, a través de las cuales se canalizan informaciones interesadas hacia los medios o se atienden sus demandas informativas. Siguien- do a Piñuel-Raigada (1997, p. 231), en la gestión de la información municipal se establecen tres funciones básicas: la mediación profesional, documentación informativa y su análisis estratégico.

\section{Las estructuras comunicativas deben} asumir la responsabilidad de articular mecanismos eficientes de gestión y evaluación de las estrategias informativas municipales

La mediación profesional representa una selección táctica de rutinas y formatos para las relaciones informativas. A través de ella, las estructuras de comunicación municipal:

- conciben, producen y difunden documentos para los periodistas:

- envían las actividades programadas por parte de los máximos representantes de la institución municipal;

atienden las demandas periodísticas;

preparan con rigor los encuentros internos como ruedas de prensa, comparecencias públicas, entrevistas o contactos directos, así como focos informativos relativos a las comisiones informativas del Pleno, las reuniones que se celebran por parte de la Comisión o Junta de Gobierno Local (en los municipios sujetos al régimen de grandes municipios), la celebración del Pleno mensual, celebración de grandes acontecimientos en el municipio, presentación de iniciativas y programas sectoriales o, incluso, la presentación de campañas institucionales de interés social.

La segunda función se centra en la documentación informativa como "la gestión de la información creada y recibida por una organización en el transcurso de sus actividades y el desarrollo de sus funciones" (Bustelo-Ruesta, 2011, p. 129). Ello implica:

- revisión diaria de los medios;

- gestión del archivo de prensa, audiovisual y digital (por temas generales o monográficos);

- producción y difusión diaria del dossier informativo para los responsables políticos de la institución municipal;

- actualización permanente de documentación básica generada por la institución municipal, así como la observación de estrategias informativas sobre temas específicos llevadas a cabo por otras instituciones, entidades, organizaciones y niveles competenciales administrativos.

El análisis de la información propicia la identificación de oportunidades para generar procesos de newsmaking a partir de dos líneas de actuación:

- estudio de las carencias o necesidades ciudadanas en cuanto a información de interés sobre la institución municipal; - análisis y evaluación de informaciones específicas (como estadísticas relacionadas con servicios, presupuestos, inversiones en infraestructuras, etc.).

El análisis de informaciones críticas permite a las estructuras comunicativas municipales el seguimiento de los asuntos conflictivos, recogiendo declaraciones e intervenciones públicas de los principales agentes implicados. Esta activi- 
dad de monitoreo orienta a los responsables institucionales para establecer argumentos de réplica que contrarresten los efectos nocivos de tales informaciones.

Esta tercera función está íntimamente interrelacionada con la segunda, de manera que el análisis estratégico informativo viene condicionado por la fase anterior de documentación sobre temas, aspectos, conflictos o situaciones que pueden derivar en consecuencias adversas para la organización municipal. Para ello, los gabinetes documentan y gestionan la información de las agendas mediáticas, a través de una codificación sistemática y rigurosa, que permite explotarla, presentarla a los responsables de la institución y actuar en consecuencia.

\section{Reciclaje de las estructuras comunicativas municipales. Formación y aprendizaje en gestión de la información}

La historia y la evolución de los gabinetes de comunicación en los ayuntamientos ha venido marcada por una constante adaptación a las estructuras municipales. La función comunicacional de las primeras instituciones democráticas, en el año 1979, se ha ido ampliando hasta llegar a configurar, en la actualidad, estructuras complejas que presentan profundas dependencias y sinergias con el resto de departamentos y áreas administrativas (Campillo-Alhama, 2010; 2011).

A las funciones tradicionales de los procesos de comunicación institucional, relaciones informativas y relaciones institucionales, se ha ido incorporando de forma progresiva el procesado de la información sobre la actividad políticoadministrativa y su documentación. Resulta muy esclarecedora la aportación de Bustelo-Ruesta (2011) cuando afirma que la integración de los procesos y controles documentales en las rutinas de trabajo representa un importante desafío para los profesionales que ejercen labores de documentación en cualquier organización.

Siguiendo a Bruce (2003), los recursos humanos integrados en las estructuras comunicativas deben ser alfabetizados para localizar y utilizar la información de los medios de comunicación de forma eficaz, y así propiciar que los responsables de la institución municipal tomen decisiones para solucionar situaciones que afectan (o pueden llegar a afectar) a los ciudadanos, basadas en la gestión del conocimiento. Dicha circunstancia supone asumir el concepto de información no solamente como proceso, sino también como producto; un producto elaborado y transformado en datos operativos que, al ser trasladados a los máximos representantes municipales, representa el reflejo fiel de la realidad mediática generada sobre un asunto municipal.

La utilización de aplicaciones informáticas diseñadas adhoc y el uso de las nuevas tecnologías en la documentación informativa se erigen como un destacado valor añadido en los procesos y rutinas periodísticas de los gabinetes de comunicación municipal. Resulta pertinente, en consecuencia, reforzar el perfil "periodístico" tradicional de funciones estandarizadas que hemos citado con anterioridad, a través de procesos de formación y aprendizaje relacionados con la documentación informativa, tal y como apuntan Wilcox, Cameron y Xifra (2006).

\section{Método e instrumento de evaluación de la difusión de políticas estratégicas municipales}

El análisis de contenido, como técnica metodológica, nos permite realizar, mediante la codificación de los soportes informativos seleccionados entre los medios de comunicación escritos offline y online, una descripción analítica de los hechos informativos a partir de diferentes variables, e interpretar los datos obtenidos a través de un proceso inferencial (Wimmer; Dominick, 1996).

A partir de los soportes, se establece un corpus de noticias para analizar la información municipal de forma fiable; corpus que podemos complementar con otros hechos municipales, bajo el formato de géneros periodísticos (informativos, interpretativos y de opinión) diferentes a la noticia.

Para ello, se propone un instrumento metodológico compuesto por 25 variables de tipo numérico, alfanumérico, fecha y lógico que adoptan valores diferentes en cada hecho informativo. Mediante un software que permite gestionar una base de datos de tales hechos, se identifican code books, traducidos en tablas que representan los valores de cada variable y que podemos relacionar vinculando unas tablas con otras. Para el proceso de codificación, se diseña un formulario para introducir texto, códigos que representan los valores y menús desplegables.

En las tablas 1.1 a 1.10 se muestran los valores utilizados para codificar las variables alfanuméricas.

Tabla 1. Variables y valores alfanuméricos del instrumento metodológico

\begin{tabular}{|c|}
\hline 1. ID (contador de noticias): tipo numérico \\
\hline 2. Número de página: tipo numérico \\
\hline 3. Fecha y día de la semana: tipo fecha \\
\hline 4. Tipos de entidad declarante: tipo alfanumérico \\
\hline 5. Tipos de declarante/s individual/es: tipo alfanumérico \\
\hline 6. Tipos de entidad declarante secundaria: tipo alfanumérico \\
\hline 7. Área organizativa. Estructura administrativa: tipo alfanumérico \\
\hline 8. Género periodístico: tipo alfanumérico \\
\hline 9. Antetítulo: tipo alfanumérico \\
\hline 10. Titular: tipo alfanumérico \\
\hline 11. Subtítulo: tipo alfanumérico \\
\hline 12. Entradilla: tipo alfanumérico \\
\hline 13. Sumario: tipo alfanumérico \\
\hline 14. Despiece: tipo lógico (sí/no) \\
\hline 15. Recuadro de apoyo: tipo lógico (sí/no) \\
\hline 16. Cintillo: tipo lógico (sí/no) \\
\hline $\begin{array}{l}\text { 17. Presentación de imagen/gráfico/foto/ilustración: tipo lógico } \\
\text { (sí/no) }\end{array}$ \\
\hline 18. Tamaño: tipo alfanumérico \\
\hline $\begin{array}{l}\text { 19. Monotemático (información simple o múltiple): tipo lógico } \\
\text { (sí/no) }\end{array}$ \\
\hline 20. Asunto o tema principal: tipo alfanumérico \\
\hline 21. Tema secundario: tipo alfanumérico \\
\hline $\begin{array}{l}\text { 22. Subtemas (contenido específico de la información): tipo } \\
\text { alfanumérico }\end{array}$ \\
\hline 23. Tratamiento de la información: tipo alfanumérico \\
\hline 24. Irrupción de la información: tipo alfanumérico \\
\hline 25. Proximidad de la información: tipo alfanumérico \\
\hline
\end{tabular}

Variables alfanuméricas de clasificación sistemática 
Tabla 1.1. Entidad declarante

\begin{tabular}{|l|l|l|}
\hline Código & Identificador_administración centralizada & Grupo \\
\hline A & Gabinete de alcaldía & ALC \\
\hline C101 & Concejalía A & C \\
\hline C102 & Concejalía B & C \\
\hline C103 & Concejalía C & C \\
\hline C104 & Concejalía D & C \\
\hline C105 & Concejalía E & C \\
\hline C106 & Concejalía F & C \\
\hline C107 & Concejalía G & C \\
\hline C108 & Concejalía H & C \\
\hline C109 & Concejalía I & C \\
\hline C110 & Concejalía J & C \\
\hline GP & Gabinete de prensa & ALC \\
\hline I & Intervención & INT \\
\hline S & Secretaría & SEC \\
\hline T & Tesorería & TES \\
\hline Código & Identificador_administración descentraliza- & Grupo \\
\hline CA & Colectivos afras entidades declarantes & \\
\hline AA & Asociaciones afectadas & COL \\
\hline AJ & Asesoría jurídica & ASO \\
\hline BIB & Biblioteca/archivo & ASE \\
\hline E101 & Empresa municipal A & VAR \\
\hline E102 & Empresa municipal B & EMU \\
\hline E201 & Empresa mixta A & EMU \\
\hline E202 & Empresa mixta B & EMI \\
\hline GP1 & Grupo político A & EMI \\
\hline GP2 & Grupo político B & GRP \\
\hline GP3 & Grupo político C & GRP \\
\hline GP4 & Grupo político D & GRP \\
\hline OA1 & Organismo autónomo 1 \\
\hline OA2 & Organismo autónomo 2 & GRP \\
\hline PA & Patronales & OA \\
\hline PL & Policía local & OA \\
\hline SI & Sindicatos & PTR \\
\hline
\end{tabular}

Tabla 1.2. Declarante individual

\begin{tabular}{|c|l|}
\hline Código & \multicolumn{1}{c|}{ Identificador } \\
\hline 0 & Ninguno \\
\hline 1 & Alcalde-presidente \\
\hline 2 & Portavoz \\
\hline 3 & Portavoz adjunto \\
\hline 4 & Concejal \\
\hline 5 & Gerente/director \\
\hline 6 & Vicepresidente \\
\hline 7 & Técnico \\
\hline 8 & Ciudadano afectado \\
\hline 9 & Representante colectivo \\
\hline 10 & Representante asociación \\
\hline 11 & Representante soporte informativo \\
\hline 12 & Inspector jefe Policía Local \\
\hline 13 & Coordinador grupo municipal \\
\hline
\end{tabular}

Tabla 1.3. Entidad declarante secundaria

\begin{tabular}{|l|l|l|}
\hline Código & Identificador_administración centralizada & Grupo \\
\hline A & Gabinete de alcaldía & ALC \\
\hline C101 & Concejalía A & C \\
\hline C102 & Concejalía B & C \\
\hline C103 & Concejalía C & C \\
\hline C104 & Concejalía D & C \\
\hline C105 & Concejalía E & C \\
\hline C106 & Concejalía F & C \\
\hline C107 & Concejalía G & C \\
\hline C108 & Concejalía H & C \\
\hline C109 & Concejalía I & C \\
\hline C110 & Concejalía J & C \\
\hline GP & Gabinete de prensa & ALC \\
\hline Código & Identificador_administración descentraliza- & Grupo \\
\hline CA & Colectivos afras entidades declarantes & \\
\hline AA & Asociaciones afectadas & COL \\
\hline AJ & Asesoría jurídica & ASO \\
\hline BIB & Biblioteca/archivo & ASE \\
\hline E101 & Empresa municipal a & VAR \\
\hline E102 & Empresa municipal b & EMU \\
\hline E103 & Empresa municipal c & EMU \\
\hline E201 & Empresa mixta a & EMU \\
\hline GP1 & Grupo político a & EMI \\
\hline GP2 & Grupo político b & GRP \\
\hline GP3 & Grupo político c & GRP \\
\hline GP4 & Grupo político d & GRP \\
\hline I & Intervención & GRP \\
\hline OA1 & Organismo autónomo 1 \\
\hline OA2 & Organismo autónomo 2 & INT \\
\hline PA & Patronales & OA \\
\hline PL & Policía local & OA \\
\hline S & Secretaría & PTR \\
\hline SI & Sindicatos & POL \\
\hline T & Tesorería & SEC \\
\hline
\end{tabular}

Tabla 1.4. Estructura administrativa

\begin{tabular}{|c|l|c|}
\hline Código & \multicolumn{1}{|c|}{ Identificador } & Legislatura $\left(\mathbf{n}^{\mathbf{0}}\right)$ \\
\hline 101 & Área administrativa 1 & -- \\
\hline 102 & Área administrativa 2 & -- \\
\hline 103 & Área administrativa 3 & -- \\
\hline 104 & Área administrativa 4 & -- \\
\hline 105 & Área administrativa 5 & -- \\
\hline 91 & Sin área & -- \\
\hline
\end{tabular}

Tabla 1.5. Género periodístico

\begin{tabular}{|c|l|}
\hline Código & \multicolumn{1}{c|}{ Identificador } \\
\hline 1 & Noticia \\
\hline 2 & Breve o reseña \\
\hline 3 & Reportaje informativo \\
\hline 4 & Entrevista objetiva \\
\hline 5 & Crónica \\
\hline 6 & Análisis \\
\hline 7 & Editorial \\
\hline 8 & Crítica \\
\hline 9 & Art. opinión o tribuna \\
\hline 10 & Columna \\
\hline 11 & Informe \\
\hline
\end{tabular}


Tabla 1.6. Tamaño o extensión

\begin{tabular}{|c|l|}
\hline Código & \multicolumn{1}{|c|}{ Identificador } \\
\hline 1 & Más de 3 páginas \\
\hline 2 & Más de 1 página \\
\hline 3 & Página completa \\
\hline 4 & Más de 1/2 página \\
\hline 5 & $1 / 2$ página \\
\hline 6 & Menos de 1/2 página \\
\hline 7 & Columna \\
\hline 8 & $1 / 2$ columna \\
\hline 9 & 1 módulo \\
\hline
\end{tabular}

Tabla 1.7. Tema principal/Tema secundario/Subtemas

\begin{tabular}{|l|l|}
\hline Código & \\
\hline APE & Aperturas \\
\hline ASE & Asesoría jurídica \\
\hline BIE & Bienestar social \\
\hline CNS & Consumo \\
\hline CON & Contratación \\
\hline COO & Cooperación al desarrollo \\
\hline CUL & Cultura \\
\hline DEP & Deporte \\
\hline EDU & Educación \\
\hline FIE & Fiestas \\
\hline FOM & Fomento \\
\hline HAC & Hacienda \\
\hline JUV & Juventud \\
\hline MED & Medio ambiente \\
\hline OMA & Oficina municipal de atención al ciudadano \\
\hline PAR & Relaciones internas partidos políticos \\
\hline PED & Pedanías y partidas rurales \\
\hline PLA & Plan estratégico \\
\hline REI & Relaciones institucionales \\
\hline REX & Relaciones externas \\
\hline RRH & Recursos humanos, organización, calidad \\
\hline SAM & Sanidad municipal \\
\hline SEG & Seguridad ciudadana \\
\hline SER & Servicios y mantenimiento \\
\hline TRA & Tráfico \\
\hline TRR & Turismo \\
\hline
\end{tabular}

Tabla 1.8. Tratamiento de la información

\begin{tabular}{|c|l|}
\hline Código & \multicolumn{1}{|c|}{ Categorización $^{1}$} \\
\hline 0 & Sin determinar \\
\hline 1 & Negativa \\
\hline 2 & Ambivalente \\
\hline 3 & Neutra \\
\hline 4 & Positiva \\
\hline
\end{tabular}

Tabla 1.9. Irrupción de la información

\begin{tabular}{|c|l|}
\hline Código & \multicolumn{1}{c|}{ Categorización $^{2}$} \\
\hline 0 & Sin determinar \\
\hline 1 & Fortuita \\
\hline 2 & Previsible \\
\hline 3 & Programada \\
\hline
\end{tabular}

Tabla 1.10. Proximidad de la información

\begin{tabular}{|c|l|}
\hline Código & \multicolumn{1}{|c|}{ Categorización $^{\mathbf{3}}$} \\
\hline 0 & Sin determinar \\
\hline 1 & Inmediata \\
\hline 2 & Mediata \\
\hline
\end{tabular}

Todas las variables y valores alfanuméricos del instrumento metodológico se integran en una base de datos (imagen 1), estableciéndose una tabla relacional que permite vincular unas con otras (imagen 2).

A través de la anterior tabla relacional, las diferentes variables quedan vinculadas y se procede a la codificación de las unidades informativas municipales que integran el corpus informativo seleccionado, utilizando un formulario (imagen 3) que permite introducir texto, códigos y seleccionar diversas opciones en los menús desplegables para realizar posteriormente el correspondiente análisis e interpretación de los datos.

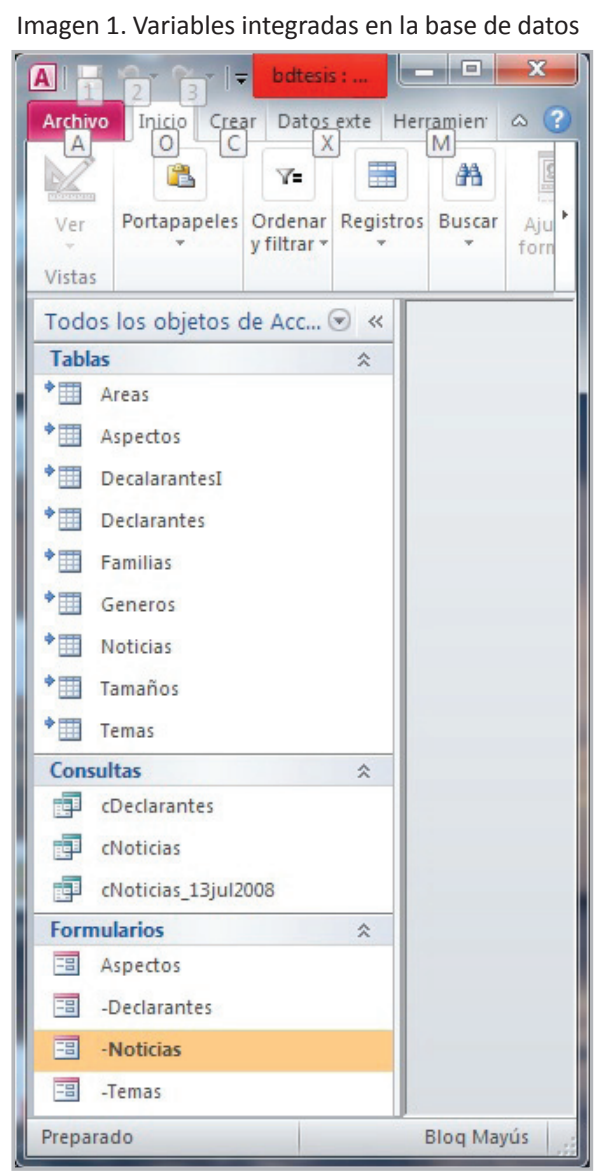

En este formulario se utilizan menús desplegables para el género periodístico, la naturaleza del tema, su irrupción, su proximidad y los subtemas específicos; los temas principales y secundarios, la entidad declarante principal, su área administrativa, el declarante individual y la entidad declarante secundaria se introducen mediante códigos; el titular, antetítulo, subtítulo, entradilla, sumario y número de página, son valores en campos no sistematizados, mientras que la fecha sirve como localizador de la unidad del corpus de noticias. 


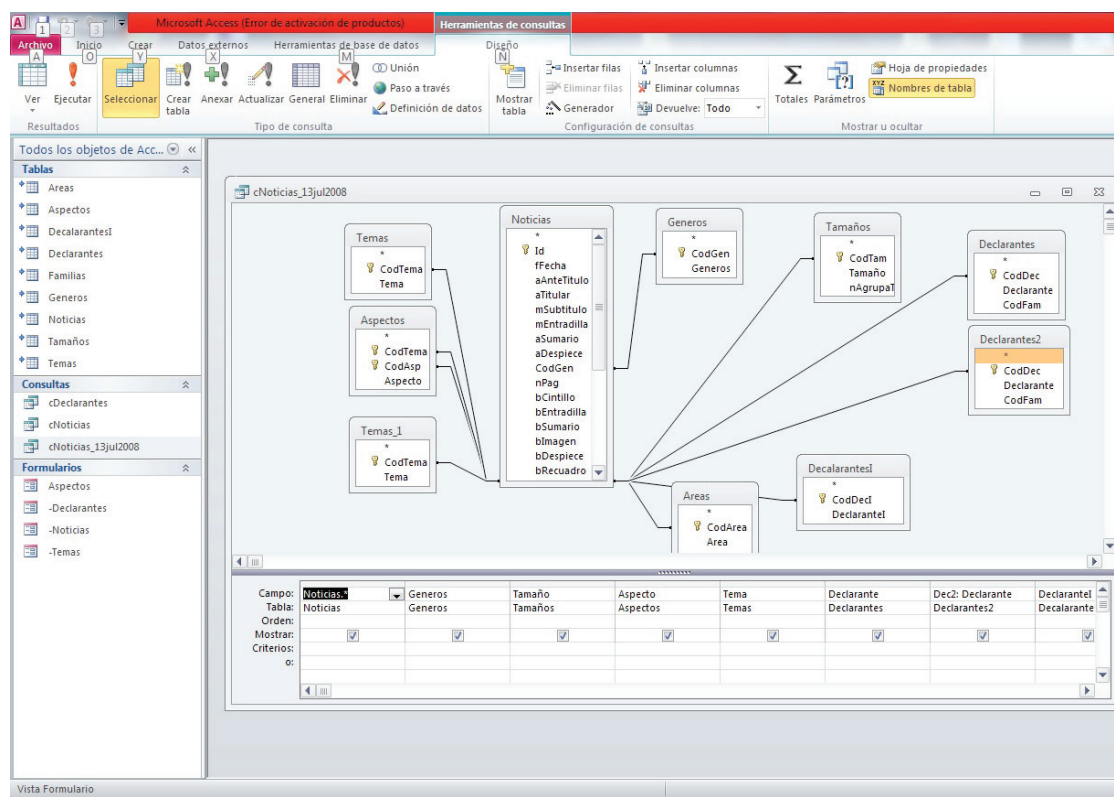

Imagen 2. Tabla relacional y consulta de selección nos, a través de los medios de comunicación social offline y online. Un seguimiento pertinente y constante del tratamiento y contextualización de los temas municipales, permitirá confirmar la idoneidad de los discursos públicos de los miembros del equipo de gobierno, la ausencia de contradicción en los mismos, así como pulsar la actuación de las fuerzas opositoras en determinados ámbitos.

Son las estructuras comunicativas las que deben asumir la responsabilidad de articular mecanismos eficientes de gestión y evaluación de las estrategias comunicativas municipales, de manera que los responsables políticos puedan tomar las oportunas decisiones, modificar o anular determinadas políticas públicas o bien explicarlas convenientemente a la ciudadanía.
Este instrumento metodológico, diseñado para medir el tratamiento y la contextualización de los temas municipales, permite la explotación e interpretación de los datos mediante un análisis descriptivo univariante y bivariante, con posibilidad de utilizar análisis multivariable en función de los resultados obtenidos.

\section{Conclusiones}

Los medios de comunicación local interpretan la realidad social y política mediante un conjunto de temas (issues) que el medio recopila con la intención de planificar, de forma genérica, su cobertura informativa. Así, la forma en que los aparatos informativos construyen la realidad social y política municipal, a partir del contenido propuesto en la agenda temática municipal, no es una operación ni inocente ni casual.

Se apunta la necesidad de que las instituciones municipales tomen conciencia de la trascendencia que pueden llegar a alcanzar determinados mensajes orientados a los ciudada-
Al obtener una radiografía de los temas básicos de la agenda mediática local y contrastarlos con encuestas de opinión, es posible pulsar la percepción de los ciudadanos respecto a la gestión municipal, y reflexionar sobre la propuesta programática del equipo de gobierno durante un período legislativo, que representa su agenda política.

\section{La utilización de aplicaciones informá- ticas diseñadas ad-hoc y el uso de las nuevas tecnologías en la documentación informativa son un valor añadido en los procesos y rutinas periodísticas de los gabinetes de comunicación municipal}

La gestión del conocimiento en los gabinetes municipales debe transformarse en un proceso mediante el cual se obtenga, organice y convierta la información elaborada por los medios de comunicación en un activo intangible, a disposición de los responsables de las instituciones municipales, con el objetivo último de asumir decisiones estratégicas vinculadas a la administración municipal. Esta circunstancia obliga a definir un nuevo perfil de los recursos humanos integrados de estas estructuras comunicativas, que van a asumir nuevas competencias como la documentación de los archivos, su sistematización y la recuperación de la información, segmentada a partir de los focos de interés de los responsables municipales.

Imagen 3. Formulario para la codificación de los datos 
Consideramos que, en el contexto actual de la sociedad de la información digital, las estructuras comunicativas municipales van a demandar profesionales de la información que sean capaces de diseñar soluciones pertinentes para el análisis y la evaluación de la información relativa a las diferentes unidades, departamentos, concejalías o áreas, a través de la implementación de modelos, instrumentos y técnicas documentales.

\section{Notas}

\section{Se identifica como}

- negativa, aquella información que distorsiona los objetivos de la organización municipal al perjudicar seriamente la imagen de la institución y sus dirigentes políticos;

- ambivalente, cuando existen en ella discursos enfrentados entre el emisor institucional y los implicados: ya sean éstos afectados directos, sus representantes o los partidos de la oposición;

- neutra, si se trata de noticias que no suponen menoscabo alguno en la gestión municipal al vincularse con la normalidad en la gestión político-administrativa;

- positiva cuando las noticias son una clara manifestación de gestión productiva y eficiente por parte de los ayuntamientos.

\section{Nos referimos a información}

- fortuita: hecho imprevisto que irrumpe intempestivamente y con cierto grado de trasgresión sobre el discurrir normal de los acontecimientos;

- previsible: hechos cuya producción o manifestación se podía predecir. Están relacionados con las rutinas, los procesos y dinámicas habituales de la gestión municipal;

- programada: acontecimientos cuyo desarrollo está previsto con antelación y cuya presentación alcanza unos índices de probabilidad muy altos. Son hechos que pueden ser excepcionales en el tiempo o que se presentan reiteradamente en períodos temporales diferentes y que obligan a preparar, con suficiente antelación, un plan de trabajo para su cobertura informativa en el momento en que tal hecho se produce.

\section{La información}

- inmediata (o de nivel 1 ) es aquella que ha sido obtenida personalmente por los informadores accediendo directamente a un hecho o documento, o bien a través de una fuente eventual o extraoficial que es protagonista causante o circunstancial del hecho;

- mediata (o de nivel 2), llega al medio de comunicación por un canal no directo: otro medio de comunicación, una fuente de información institucional (gabinete de prensa) o estable (agencia informativa) que las había obtenido de forma inmediata a través de fuentes de primer nivel o bien a través de personas que no han sido testigos directos de los hechos acontecidos.

\section{Bibliografía}

Almansa-Martínez, Ana (2011). Del gabinete de prensa al gabinete de comunicación. Madrid: Comunicación Social.

\section{ISBN: 9788492860906}

Bruce, Christine-Susan (2003). "Las siete caras de la alfabetización en información en la enseñanza superior". Anales de documentación, n. 6, pp. 289-294.

http://revistas.um.es/index.php/analesdoc/article/viewFile/3761/3661

Bustelo-Ruesta, Carlota (2011). "Los grandes temas relacionados con la gestión de documentos: desafíos y oportunidades". El profesional de la información, marzo-abril, v. 20, n. 2, pp. 129-133.

http://eprints.rclis.org/15627

http://dx.doi.org/10.3145/epi.2011.mar.01

Campillo-Alhama, Concepción (2010). “Comunicación pública y administración municipal. Una propuesta de modelo estructural". Pensar la publicidad, v. IV, n. 1, pp. 45-62. http://revistas.ucm.es/index.php/PEPU/article/view/ PEPU1010120045A

Campillo-Alhama, Concepción (2011). Comunicación pública y gestión estratégica municipal. Un estudio exploratorio sobre la agenda temática. Colección Premios Blas Infante 2010. Sevilla: Instituto Andaluz de Hacienda y Administración Pública. ISBN: 9788483335598

Campillo-Alhama, Concepción; Ramos-Soler, Irene (2013). "La comunicación 2.0 de las políticas orientadas a mayores en los ayuntamientos españoles". Estudios sobre el mensaje periodístico, v. 19, n. especial abril, pp. 661-670.

http://revistas.ucm.es/index.php/ESMP/article/view/42149 http://dx.doi.org/10.5209/rev_ESMP.2013.v19.42149

Cebrián, Mariano (2012). Periodismo empresarial e institucional. Madrid: Comunicación Social. ISBN: 9788415544081

Edo, Concha (2009). Periodismo informativo e interpretativo. El impacto de internet en la noticia, las fuentes y los géneros. Madrid: Comunicación Social. ISBN: 9788496082960

Galán-Galán, Alfredo (2000). "Comunicación pública”. En: Tornos, Joaquín; Galán, Alfredo (coords.). La comunicación pública: información administrativa al ciudadano. Madrid: Marcial Pons, pp. 23-146. ISBN: 8472488241

Piñuel-Raigada, José-Luis (1997). Teoría de la comunicación y gestión de las organizaciones. Madrid: Síntesis. ISBN: 84 77384908

Wilcox, Dennis L.; Cameron, Glen T.; Xifra, Jordi (2006). Relaciones públicas: estrategias y tácticas. Madrid: Pearson. ISBN: 9788420550353

Wimmer, Roger D.; Dominick, Joseph R. (1996). La investigación científica de los medios de comunicación. Una introducción a sus métodos. Barcelona. Colección Bosch Comunicación. ISBN: 9788476763599

Xifra, Jordi (2011). "Subsidios informativos y función documental de las salas de prensa online de los ministerios españoles". El profesional de la información, mayo-junio, v. 20, n. 3, pp. 270-275.

http://dx.doi.org/10.3145/epi.2011.may.04 